\title{
The Transfer of Large-scale Magnetic Field by Radial Inhomogeneity of the Material Density in the Rotating Convection Zone
}

\author{
V.N. Krivodubskij ${ }^{1}$, L.L. Kichatinov ${ }^{2}$ \\ ${ }^{1}$ Kiev University Astronomical Observatory, Observatorna Street 3, \\ Kiev - 53, 252053, Ukraine, USSR \\ ${ }^{2}$ Sib IZMIR, P.O. Box 4026, Irkutsk - 33, 664033, Russia, USSR
}

\begin{abstract}
The influence of rotation on the transfer of the mean magnetic field of the Sun, caused by the radial inhomogeneity of the solar turbulent plasma density, is investigated. It turns out that the transfer directions of the poloidal and toroidal magnetic fields do not coincide.
\end{abstract}

Random turbulent hydrodynamic motions (with velocities $u^{\prime}$ ) in a medium of high conductivity generate random fluctuating magnetic fields $\left(h^{\prime}\right)$. Such small-scale magnetic fluctuations cause the mean large-scale magnetic field to rise with speed proportional to the density gradient of the medium $(\nabla Q)$ (Kichatinov, 1982). In the nonlinear formulation of the problem the magnetic field transfer takes place in the direction of increasing plasma density, independent of the magnetic field configuration (Kichatinov, 1982; Vainshtein, 1983). The expulsion velocity of the horizontal large-scale magnetic field of the Sun in the convective zone has been calculated by Krivodubskij (1987). Here the calculation of the transfer effect of the solar magnetic field is carried out for a convection zone with density stratification, taking into account the anisotropy in the turbulence generated by the solar rotation.

The calculation of the mean electromotive force in the turbulent medium is carried out in the quasilinear approximation under the condition of equipartition of the kinetic and magnetic energies of small-scale fluctuations (Kichatinov, 1990). It is found that the transfer directions of poloidal $\left(V^{a}\right)$ and toroidal $\left(V^{b}\right)$ magnetic fields do not coincide in the highly turbulent rotating convective envelope of the Sun. We write

$$
\begin{aligned}
& V^{a}=-V+V_{m}+V_{m}^{\prime}, \\
& V^{b}=+V+V_{m},
\end{aligned}
$$

where $\boldsymbol{V}$ is the transfer velocity of the mean magnetic field caused by the turbulent motions (the kinematic contribution); $V_{m}$ is the transfer velocity of the mean field caused by the small-scale magnetic fluctuations (the nonlinear contribution); $\boldsymbol{V}_{m}^{\prime}$ 
is the additional transfer velocity of the poloidal magnetic field (resulting from rotational and magnetic fluctuations). These components of the transfer velocity are given by

$$
\begin{aligned}
V & =-\boldsymbol{\lambda}_{\perp} \tau u^{\prime 2} \Phi_{1}(\beta), \\
\boldsymbol{V}_{m} & =+\lambda \tau u^{\prime 2} \Phi_{2}(\beta), \\
\boldsymbol{V}_{m}^{\prime} & =-\boldsymbol{\lambda}_{\perp} \tau u^{\prime 2} \Phi_{3}(\beta),
\end{aligned}
$$

where $\lambda=\nabla Q / Q ; \tau \simeq l / u^{\prime 2}$ is the timescale of the nonlinear relaxation of fluctuations which is equal, to order of magnitudes, to the correlation time; $l$ is the characteristic size of small-scale velocity $\left(u^{\prime}\right)$ and magnetic field $\left(h^{\prime}\right)$ fluctuations; $\Phi_{n}(\beta)$ are functions of the Coriolis number $\beta=2 \tau \Omega ; \Omega$ is the angular velocity.

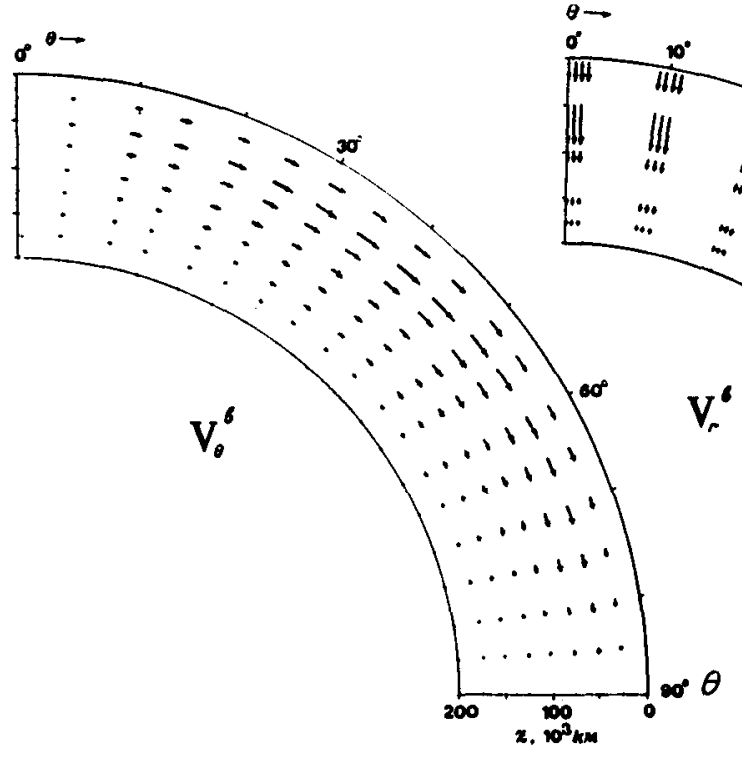

Fig. 1.
Fig. 2.

The expressions for the meridional $(\theta)$ and radial $(r)$ transfer velocity components of poloidal $\left(V^{a}, V_{r}^{a}\right)$ and toroidal $\left(V^{b}, V_{r}^{b}\right)$ large-scale magnetic fields in the usual spherical coordinate system $r, \theta, \phi(\theta$ is the co-latitude) take the form (Kichatinov, 1990) 


$$
\begin{aligned}
V^{a} & =-\frac{3 \kappa g}{(\gamma-1) C_{p} T} \cos \theta \sin \theta\left[\Phi_{1}(\beta)-\Phi_{3}(\beta)\right], \\
V_{r}^{a} & =-\frac{3 \kappa g}{(\gamma-1) C_{p} T}\left\{\Phi_{2}(\beta)+\sin ^{2} \theta\left[\Phi_{1}(\beta)-\Phi_{3}(\beta)\right]\right\}, \\
V^{b} & =+\frac{3 \kappa g}{(\gamma-1) C_{p} T} \cos \theta \sin \theta \Phi_{1}(\beta), \\
V_{r}^{b} & =-\frac{3 \kappa g}{(\gamma-1) C_{p} T}\left[\Phi_{2}(\beta)-\Phi_{1}(\beta) \sin ^{2} \theta\right],
\end{aligned}
$$

where $\kappa \simeq \frac{1}{3} \tau u^{\prime 2}$ is the turbulent diffusion coefficient, $C_{p}$ is the specific heat capacity at constant pressure, $T$ is the temperature, $g$ is the acceleration due to gravity and $\gamma$ is the adiabatic index.

The values of the different components of the transfer velocities of the magnetic fields for the convection zone model of Spruit (1977) are calculated. The poloidal field is transferred deep into the convection zone (the maximum transfer velocity $V_{r}^{a}$ is about $\left.2 \times 10^{3} \mathrm{~cm} / \mathrm{s}\right)$ and mainly to the pole $\left(V_{\theta \max }^{a} \simeq 10^{2} \mathrm{~cm} / \mathrm{s}\right)$. The horizontal component of the toroidal field $V_{\theta}^{b}$ is directed to the equator $\left(V_{\theta \max }^{b} \simeq 6 \times 10^{2}\right.$ $\mathrm{cm} / \mathrm{s}$ ) (Fig. 1), in accordance with the observed picture of the active region migrations.

The radial component of this velocity $\left(V_{r}^{b}\right)$ depends on the latitude (Fig. 2). At high latitudes it is directed inwards $\left(V_{r \max }^{b} \simeq-2 \times 10^{3} \mathrm{~cm} / \mathrm{s}\right)$ whilst near the equator it is directed towards the surface $\left(V_{r \max }^{b} \simeq+2 \times 10^{2} \mathrm{~cm} / \mathrm{s}\right)$, with the exception of the upper layers of the convection zone where it is directed deep into the Sun. Therefore, the toroidal magnetic fields in the near-pole regions may be supposed to be blocked in deep layers of the convective zone, due to the transfer mechanism considered. In the near-equator region the toroidal fields in the lower part of the convection zone migrate upward reaching levels where the magnetic buoyancy starts to play the essential role (i.e. the effect of buoyancy exceeds the effect of blocking) and as a result it causes the active regions to rise.

\section{References}

Kichatinov, L.L.: 1982, Magnitnaya Gidrodinamika 2, 67

Kichatinov, L.L.: 1990, Astron. Astrophys. (in press)

Krivodubskij, V.N.: 1987, Pis'ma Astron. Zh. 9, 803 (Sov. Astron. Lett. 9, 338)

Spruit, H.C.: 1977, Thesis, University of Utrecht, p.17

Vainshtein S.I.: 1983, Magnetic Fields in Outer Space (in Russian), Nauka, Moscow 